Pacific

Journal of

Mathematics

\title{
DISTORTION OF WREATH PRODUCTS IN SOME FINITELY PRESENTED GROUPS
}

SEAN Cleary 


\title{
DISTORTION OF WREATH PRODUCTS IN SOME FINITELY PRESENTED GROUPS
}

\author{
SEAN CLEARY
}

\begin{abstract}
Wreath products such as $\mathbb{Z} z \mathbb{Z}$ are not finitely presentable yet can occur as subgroups of finitely presented groups. Here we compute the distortion of $\mathbb{Z} Z \mathbb{Z}$ as a subgroup of Thompson's group $F$ and as a subgroup of Baumslag's metabelian group $G$. We find that $\mathbb{Z} z \mathbb{Z}$ is undistorted in $F$ but is at least exponentially distorted in $G$.
\end{abstract}

\section{Introduction}

We consider aspects of the question of the distortion of infinitely related groups as subgroups of finitely presented groups. Higman [1961] showed that every recursively presentable group occurs as a subgroup of a finitely presented group, but it is not clear in general what happens to the geometry of the group since this embedding uses complicated algebraic methods and methods from recursive function theory which may affect the geometry of the group severely. Ol'shanskir [1997] constructed isometric embeddings of recursively presentable groups into finitely presented groups using difficult methods that do not lead to easily constructed examples. In the particular concrete cases here, we consider concrete embeddings of one of the simplest finitely generated but not finitely presentable groups, $\mathbb{Z} \imath \mathbb{Z}$. We consider two embeddings of $\mathbb{Z} \imath \mathbb{Z}$ into finitely presented groups. The first is as a subgroup of Thompson's group $F$ and the second is as subgroup of Baumslag's remarkable finitely presented metabelian group which contains $\mathbb{Z} \imath \mathbb{Z}$ and thus a free abelian subgroup of infinite rank. The distortion of the metric of $\mathbb{Z} \imath \mathbb{Z}$ is linear in Thompson's group $F$ but is at least exponential in Baumslag's group.

\section{Background}

Metrics of wreath products. Two of the simplest infinite wreath products are the lamplighter group $\mathbb{Z}_{2} \imath \mathbb{Z}$ and $\mathbb{Z} \imath \mathbb{Z}$. Cleary and Taback [2005] analyzed aspects of

MSC2000: 20F65.

Keywords: subgroup distortion, Thompson's group.

The author gratefully acknowledges support from PSC-CUNY grant \#64459-0033 and the hospitality of the Centre de Recerca Matemàtica. 
the metric geometry of those groups and other wreath products. There are natural normal forms for elements in these groups which lead to geodesic words for elements in these groups with respect to their standard generating sets.

For $\mathbb{Z} \imath \mathbb{Z}$, we consider the standard presentation

$$
\left.\langle a, t|\left[a^{t^{i}}, a^{t^{j}}\right], \text { for } i, j \in \mathbb{Z}\right\rangle,
$$

where $a^{b}$ denotes the conjugate $b^{-1} a b$ and $[a, b]$ the commutator $a b a^{-1} b^{-1}$.

Geometrically, we can think of this wreath product by imagining a string of counters arranged from left to right and infinite in both directions, with one counter distinguished as the origin. As in the lamplighter group, we imagine a cursor that moves along the string of counters and will point to a particular one of these counters as being of current interest. The generator $a$ acts as a generator of $\mathbb{Z}$ in the factor to which the cursor currently points and increases the counter in that factor, and the generator $t$ moves the cursor to the right to the next counter. A typical such word is illustrated in Figure 1.

The starting configuration of these counters, corresponding to the identity element in $\mathbb{Z} Z \mathbb{Z}$, is with all of the counters at zero and the cursor resting at the counter designated at the origin. We consider a word in these generators as a sequence of instructions to move the cursor and change the counter in the current factor. After application of a long string of the generators, we will be in a state where a finite number of counters are nonzero and the cursor points at a particular counter, called the final position of the cursor for that word.

We define $a_{n}=a^{t^{n}}$ and note that $a_{n}$ is a generator of the conjugate copy of $\mathbb{Z}$ indexed by $n$. These $a_{n}$ commute and, as described in [Cleary and Taback 2005], we can put any word in the generators into one of two normal forms:

$$
\begin{aligned}
& r f(w)=a_{i_{1}}^{e_{1}} a_{i_{2}}^{e_{2}} \ldots a_{i_{k}}^{e_{k}} a_{-j_{1}}^{f_{1}} a_{-j_{2}}^{f_{2}} \ldots a_{-j_{l}}^{f_{l}} t^{m} \quad \text { (right-first), } \\
& l f(w)=a_{-j_{1}}^{f_{1}} a_{-j_{2}}^{f_{2}} \ldots a_{-j_{l}}^{f_{l}} a_{i_{1}}^{e_{i}} a_{i_{2}}^{e_{i}} \ldots a_{i_{k}}^{e_{k}} t^{m} \quad \text { (left-first), }
\end{aligned}
$$

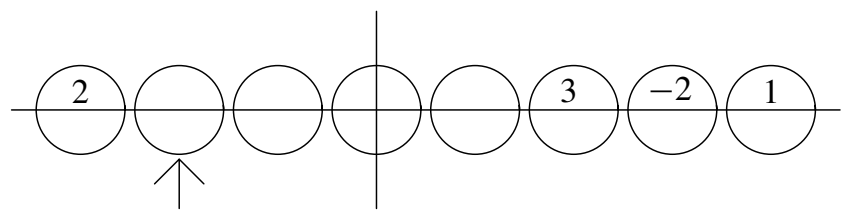

Figure 1. Diagram for $w=a_{2}^{3} a_{3}^{-2} a_{4} a_{-3}^{2} t^{-2}$. The origin in the wreath product direction is indicated by a vertical line, empty circles denote counters which are zero, and the final cursor position is indicated by the arrow. 
with $i_{k}>\ldots i_{2}>i_{1} \geq 0$ and $j_{l}>\ldots j_{2}>j_{1}>0$ and $e_{i}, f_{j} \neq 0$.

The final resting position of the cursor is easily seen to be $m$ for either of these normal forms, and we can see that the leftmost nonzero counter is in position $-j_{l}$ and the rightmost nonzero counter is in position $i_{k}$.

In the right-first form, $r f(w)$, the cursor moves first to the right from the origin, changing the counters in the appropriate factors as the cursor moves to the right. Then the cursor moves back to the origin not affecting any of the counters until passing the origin. Past the origin, the cursor continues to work leftwards, again changing the counters in the appropriate factors. Finally, the cursor moves to its ending location from the leftmost nonzero counter to the left of the origin.

The left-first form is similar, but instead of initially moving to the right, the cursor begins by moving toward the left.

At least one of these normal forms will lead to minimal-length representation for $w$, depending upon the final location of the cursor. If $m$ is nonnegative, then the leftfirst normal form will lead to a geodesic representative, and if $m$ is nonpositive, the right-first normal form will lead to a geodesic representative, giving the following measurement of length:

Proposition 2.1 [Cleary and Taback 2005, Proposition 3.8]. If a word $w \in \mathbb{Z} \imath \mathbb{Z}$ is in either right-first or left-first normal form, the word length of $w$ with respect to $\{a, t\}$ satisfies

$$
|w|=\sum_{n=1}^{k}\left|e_{i_{n}}\right|+\sum_{n=1}^{l}\left|f_{j_{n}}\right|+\min \left\{2 j_{l}+i_{k}+\left|m-i_{k}\right|, 2 i_{k}+j_{l}+\left|m+j_{l}\right|\right\} .
$$

The first two terms are the minimum number of applications of $a^{ \pm 1}$ needed to put all of the counters into their desired states and the last term is the minimum amount of movement required to visit the leftmost and rightmost nonzero counters and then the final position of the cursor, counting the required applications of $t^{ \pm 1}$.

The word $w=a_{2}^{3} a_{3}^{-2} a_{4} a_{-3}^{2} t^{-2}$ pictured in Figure 1 has geodesic representatives in right-first normal form since the final position of the cursor is to the left of the origin. One such minimal length representative is $t^{2} a^{3} t a^{-2} t a t^{-7} a^{2} t$, of length 20 .

\section{3. $\mathbb{Z} Z \mathbb{Z}$ as a subgroup of Thompson's group $F$}

Thompson's group $F$ is a remarkable finitely generated, finitely presented group that can be understood via a wide range of perspectives. For an excellent overview of its properties, see [Cannon et al. 1996]. The standard infinite presentation of $F$ is

$$
\left.\left\langle x_{0}, x_{1}, \ldots\right| x_{n}^{x_{i}}=x_{n+1} \text { for } i<n\right\rangle .
$$

Since $x_{2}=x_{1}^{x_{0}}$ and so on, $F$ is generated by the first two generators and we can define $x_{n+1}=x_{n}^{x_{0}}$ to express all generators and thus all group elements in terms of 
$x_{0}$ and $x_{1}$. Furthermore, all of these infinitely many relations are consequences of the first two nontrivial relations, so we have the standard finite presentation

$$
\left\langle x_{0}, x_{1} \mid x_{2}^{x_{1}}=x_{3}, x_{3}^{x_{1}}=x_{4}\right\rangle .
$$

Thompson's group $F$ can be described in terms of rooted tree pair diagrams, and there is a straightforward method of converting between words in a normal form with respect to the infinite generating set and tree pair diagrams, via the method of leaf exponents, as described in [Cannon et al. 1996]. There is also an easy method of converting from tree pair diagrams to piecewise-linear homeomorphisms of the unit interval where $F$ can be regarded as the subgroup of elements with dyadic breakpoints and slopes which are powers of 2 . There is a natural notion of a reduced tree pair diagram described there and there are efficient means to convert between the unique normal form for an element of $F$ and the unique reduced tree pair diagram for that word.

We consider a rooted binary tree with $n$ leaves as being constructed of $n-1$ carets, which are interior nodes of the tree together with the two downward directed edges from that node. The left side of a tree consists of nodes and edges connected to the root by a path consisting only of left edges, and similarly for the right side A tree pair diagram $(S, T)$ is made up of a "positive" tree $T$ and a "negative" tree $S$ with the same number of leaves.

To understand the metric properties of $F$, we consider expressing words with respect to the finite generating set. Burillo, Cleary and Stein [2001] estimated the word length in terms of the number of carets and showed that the number of carets is quasiisometric to the word length. Fordham [2003] developed a remarkable method using tree pair diagrams to efficiently compute exact word length and find minimal length representatives of words.

We can understand word length of elements represented as tree pair diagrams by understanding how the generators change the tree pair diagram for $w$ to that for $w g$ for the generators, as described in [Fordham 2003; Cleary and Taback 2004]. The right actions of the generators can be described as 'rotations' which change the negative tree in a possibly unreduced representative of the element.

The wreath product $\mathbb{Z} \imath \mathbb{Z}$ is a subgroup of $F$ and can be realized in many different ways. Perhaps the simplest is as the subgroup $H$ generated by $x_{0}$ and $h=x_{1} x_{2} x_{1}^{-2}$, pictured in Figure 2. The element $h$, regarded as a piecewise-linear homeomorphism of the unit interval, has support $\left[\frac{1}{2}, \frac{3}{4}\right]$. The conjugates $h^{x_{0}^{n}}$ have support

$$
\begin{array}{r}
{\left[\frac{2^{n+1}-1}{2^{n+1}}, \frac{2^{n+2}-1}{2^{n+2}}\right] \text { for } n \geq 0,} \\
{\left[\frac{1}{2^{1-n}}, \frac{1}{2^{-n}}\right] \text { for } n<0 .}
\end{array}
$$



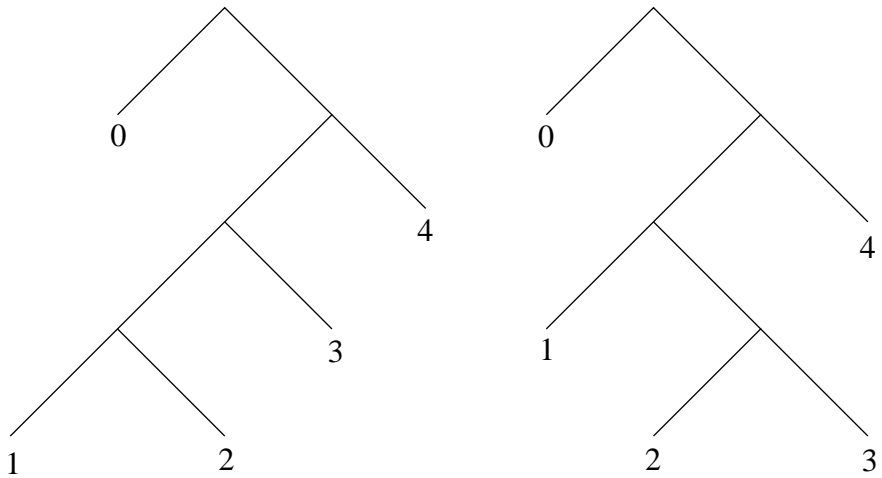

Figure 2. Tree pair diagram for $x_{1} x_{2} x_{1}^{-2}$, the image of $a$ under $\phi$.

The isomorphism between this subgroup and $\mathbb{Z} Z \mathbb{Z}$ is given by the homomorphism $\phi(w): \mathbb{Z} \imath \mathbb{Z} \rightarrow F$ where $\phi(a)=h=x_{1} x_{2} x_{1}^{-2}$ and $\phi(t)=x_{0}$. The conjugates of $\phi(a)$ by $x_{0}$ generate infinite cyclic groups, and these conjugates each have interiors of their supports which are disjoint from the interiors of the supports of the other conjugates. Thus they freely generate a free abelian group of countable rank. Since $x_{0}$ conjugates each of these abelian factors to the next, the isomorphism is readily established with $\mathbb{Z} 2 \mathbb{Z}$.

To understand the distortion of the subgroup $H$ in $F$, we compare the word length of an element $w=a_{i_{1}}^{e_{1}} a_{i_{2}}^{e_{2}} \ldots a_{i_{k}}^{e_{k}} a_{-j_{1}}^{f_{1}} a_{-j_{2}}^{f_{2}} \ldots a_{-j_{l}}^{f_{l}} t^{m}$ with its image in $F$.

Theorem 3.1. The subgroup $H$ isomorphic to $\mathbb{Z} \imath \mathbb{Z}$ in $F$ generated by $x_{0}=\phi(t)$ and $h=x_{1}^{2} x_{2}^{-1} x_{1}^{-1}=\phi(a)$ is undistorted.

Proof. We count the number of carets of the image of a word $w$. First, we consider the case when $m=0$ and then the cases where $m$ is nonzero.

Case $m=0$. Here, the image of the word as a tree pair diagram has a characteristic form where the root of the positive tree is paired with the root of the negative tree, such as that shown in Figure 3. In the general case, where both $k$ and $l$ are positive, we have

- a single root caret,

- $i_{k}+1$ right carets,

- $\sum_{n=1}^{k}\left(\left|e_{n}\right|+1\right)$ interior carets below the right arm of the tree,

- $j_{l}$ left carets, and

- $\sum_{n=1}^{l}\left(\left|f_{n}\right|+1\right)$ interior carets below the left arm of the tree. 

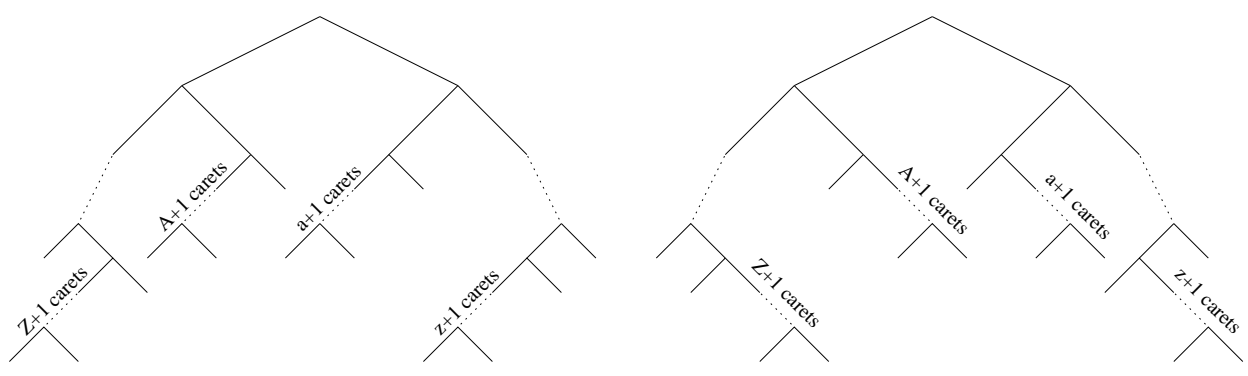

Figure 3. The tree pair diagram for the image $\phi(w)$ of a word $w=$ $a_{0}^{a} a_{1}^{b} \ldots a_{n}^{z} a_{-1}^{A} a_{-2}^{B} \ldots a_{-m}^{Z}$ with $t$ exponent sum 0 and all positive exponents for $a_{i}$.

This gives a total of

$$
N(\phi(w))=i_{k}+j_{l}+2+\sum_{n=1}^{k}\left(\left|e_{n}\right|+1\right)+\sum_{n=1}^{l}\left(\left|f_{n}\right|+1\right)
$$

carets in the image of $w$. By [Burillo et al. 2001], the number of carets is quasiisometric to the word length in $F$ with respect to $\left\{x_{0}, x_{1}\right\}$ and since the length of $w$ in $\mathbb{Z} \imath \mathbb{Z}$ is

$$
2 j_{l}+2 i_{k}+\sum_{n=1}^{k}\left|e_{n}\right|+\sum_{n=1}^{l}\left|f_{n}\right|
$$

we see that these lengths are quasiisometric.

The image of a typical word with all $e_{n}$ and $f_{n}$ positive is shown in Figure 3, corresponding to a series of rightward rotations at nodes distance one from the sides of the tree.

Case $m>0$. Here we start with the same tree pair diagram for the $m=0$ case and apply $x_{0}$ on the right $m$ times. Each application of $x_{0}$ will change the negative tree by moving the root caret to a right caret and the topmost left caret to the root, if there is a left caret. If there is no left caret, a new caret will need to be added for each such application. For each application of $x_{0}$ which requires a new caret, in the negative tree, that new caret will become the root caret and in the positive tree, the new caret will be added as the left child of the leftmost caret. Since there are $j_{l}$ left carets, if $m \leq j_{l}$, we do not need to add any carets and the number of carets is

$$
i_{k}+j_{l}+2+\sum_{n=1}^{k}\left(\left|e_{n}\right|+1\right)+\sum_{n=1}^{l}\left(\left|f_{n}\right|+1\right)
$$

as before. If $m>j_{l}$, we will need to add $m-j_{l}$ new carets and will have

$$
i_{k}+j_{l}+2+\sum_{n=1}^{k}\left(\left|e_{n}\right|+1\right)+\sum_{n=1}^{l}\left(\left|f_{n}\right|+1\right)+m-j_{l}
$$


carets. Again, these quantities give lengths which are comparable to word length in $\mathbb{Z} \imath \mathbb{Z}$.

Case $m<0$. This works in the same way as the case $m>0$.

Thus in all cases $\phi$ does not distort distances more than linearly, so the subgroup $H$ isomorphic to $\mathbb{Z} \imath \mathbb{Z}$ is undistorted in $F$.

We can obtain more precise estimates of the quasiisometry constants using Fordham's method [2003] for computing exact lengths in $F$. We can keep track of the particular caret pairings and their weights and we find that the caret pairings that occur are easily computed. Caret pairing types are described in [Fordham 2003; Cleary and Taback 2004]. For example, in the case where $m=0$ and both $l$ and $k$ are positive, we have the following caret pairs:

- One caret pair of type $\left(L_{0}, L_{0}\right)$ from the leftmost carets, contributing no weight.

- $j_{l}$ caret pairs of type $\left(L_{L}, L_{L}\right)$ from the left side and root, contributing weight $2 j_{l}$.

- $i_{k}-1$ caret pairs of types $\left(R_{*}, R_{*}\right)$ not of type $\left(R_{0}, R_{0}\right)$, contributing weight $2\left(i_{k}-1\right)$.

- One caret pair of type $\left(R_{0}, R_{0}\right)$ from the rightmost carets, contributing no weight.

- For each $e_{n}>0$, there will be a single pairing of type $\left(I_{0}, I_{0}\right)$ contributing weight 2 and $e_{n}-1$ pairings of type $\left(I_{0}, I_{R}\right)$, contributing weight $4\left(e_{n}-1\right)$.

- For each $e_{n}<0$, there will be a single pairing of type $\left(I_{0}, I_{0}\right)$ contributing weight 2 and $\left|e_{n}\right|-1$ pairings of type $\left(I_{R}, I_{0}\right)$, contributing weight $4\left(\left|e_{n}\right|-1\right)$.

- Similarly, for the interior carets from the left side of the tree, we have for each $f_{n}$, there will be a single pairing of type $\left(I_{0}, I_{0}\right)$ contributing weight 2 and $\left|f_{n}\right|-1$ pairings of type $\left(I_{0}, I_{R}\right)$ or $\left(I_{R}, I_{0}\right)$, contributing weight $4\left(\left|f_{n}\right|-1\right)$.

These will give a total weight of

$$
\begin{aligned}
2 j_{l}+2\left(i_{k}-1\right)+2 k+4 \sum\left|e_{n}\right|+ & 2 l+4 \sum\left|f_{n}\right| \\
& =2 j_{l}+2 i_{k}+2 k+2 l+4 \sum\left|e_{n}\right|+4 \sum\left|f_{n}\right|-2
\end{aligned}
$$

in the case when $m=0$, which compares to the corresponding length in $\mathbb{Z} \imath \mathbb{Z}$ of $2 j_{l}+2 i_{k}+\sum\left|e_{n}\right|+\sum\left|f_{n}\right|$.

Again, these give lengths comparable to word length in $\mathbb{Z} \imath \mathbb{Z}$. After a similar analysis for other cases, we see that for a word $w$ in $\mathbb{Z} \imath \mathbb{Z}$, we have

$$
|w|_{\mathbb{Z} Z \mathbb{Z}}-2 \leq|\phi(w)|_{F} \leq 4|w|_{\mathbb{Z} Z \mathbb{Z}} .
$$




\section{4. $\mathbb{Z} z \mathbb{Z}$ as a subgroup of Baumslag's metabelian group}

Baumslag [1972] introduced the group $G=\left\langle a, s, t \mid[s, t],\left[a^{t}, a\right], a^{s}=a a^{t}\right\rangle$ to show that a finitely presented metabelian group can contain free abelian subgroups of infinite rank. This group in fact contains $\mathbb{Z} \imath \mathbb{Z}$ : all relators of the form $\left[a^{t^{i}}, a^{t^{j}}\right]$ are consequences of these three, so the subgroup $H$ generated by $a$ and $t$ is isomorphic to $\mathbb{Z} \imath \mathbb{Z}$.

Here we examine the distortion of this subgroup in $G$.

Theorem 4.1. The subgroup $H$ has at least exponential distortion in $G$.

Proof. First, $s$ conjugates elements in $H$ to other elements in $H$ in a manner illustrated here:

$$
a^{\left(s^{2}\right)}=\left(a^{s}\right)^{s}=\left(a a^{t}\right)^{s}=a^{s}\left(a^{s}\right)^{t}=a a^{t}\left(a a^{t}\right)^{t}=a a^{t} a^{t} a^{t^{2}}=a_{0} a_{1}^{2} a_{2} .
$$

In terms of the notation described above, we have $a_{n}^{s}=a_{n} a_{n+1}$. Further conjugation by $s$ leads to increasingly long words, such as

$$
a^{\left(s^{3}\right)}=\left(a_{0} a_{1}^{2} a_{2}\right)^{s}=a_{0} a_{1} a_{1}^{2} a_{2}^{2} a_{2} a_{3}=a_{0} a_{1}^{3} a_{2}^{3} a_{3},
$$

and we notice the occurrence of the binomial coefficients with repeated iteration, formalized below:

Lemma 4.2. Higher conjugates of a by $s$ in $G$ give elements of the following form:

$$
a^{s^{n}}=a_{0}^{\left(\begin{array}{c}
n \\
0
\end{array}\right)} a_{1}^{\left(\begin{array}{c}
n \\
1
\end{array}\right)} \ldots a_{n}^{\left(\begin{array}{c}
n \\
n
\end{array}\right)} .
$$

Proof. We work by induction. The cases with $n=1,2$ and 3 are described above, and by assuming it is true for $n$ we derive

$$
a^{s^{n+1}}=\left(a_{0}^{\left(\begin{array}{c}
n \\
0
\end{array}\right)} a_{1}^{\left(\begin{array}{c}
n \\
1
\end{array}\right)} \ldots a_{n}^{\left(\begin{array}{c}
n \\
n
\end{array}\right)}\right)^{s}=a_{0}^{\left(\begin{array}{c}
n \\
0
\end{array}\right)} a_{1}^{\left(\begin{array}{c}
n \\
0
\end{array}\right)} a_{1}^{\left(\begin{array}{c}
n \\
1
\end{array}\right)} a_{2}^{\left(\begin{array}{c}
n \\
1
\end{array}\right)} \ldots a_{n}^{\left(\begin{array}{c}
n \\
n
\end{array}\right)} a_{n+1}^{\left(\begin{array}{c}
n \\
n
\end{array}\right)}=a_{0}^{\left(\begin{array}{c}
n+1 \\
0
\end{array}\right)} \ldots a_{n+1}^{\left(\begin{array}{c}
n+1 \\
n+1
\end{array}\right)},
$$

using the commutativity of the $a_{i}$ and the fact that $a_{i}^{s}=a_{i} a_{i+1}$.

Returning to the proof of the theorem, we see that $a^{s^{n}}$ has length $2 n+1$ as an element of $G$, and that it lies in the subgroup $H$, as there is a representative with no occurrences of $s$.

To compute the length of this element in the subgroup $H$ with respect to its generators $a$ and $t$, we use the method described in Section 2 on the expression with the binomial coefficients and find that

$$
\left|a^{s^{n}}\right|_{H}=2 n+\sum_{i=0}^{n}\left(\begin{array}{l}
n \\
i
\end{array}\right)=2 n+2^{n} .
$$

Thus we have $\left|a^{s^{n}}\right|_{H}=2 n+2^{n}$ while $\left|a^{s^{n}}\right|_{G}=2 n+1$, so the wreath product $\mathbb{Z} \imath \mathbb{Z}$ is at least exponentially distorted in $G$. 


\title{
References
}

[Baumslag 1972] G. Baumslag, "A finitely presented metabelian group with a free abelian derived group of infinite rank”, Proc. Amer. Math. Soc. 35 (1972), 61-62. MR 45 \#8710 Zbl 0269.20029

[Burillo et al. 2001] J. Burillo, S. Cleary, and M. I. Stein, "Metrics and embeddings of generalizations of Thompson's group F", Trans. Amer. Math. Soc. 353:4 (2001), 1677-89. MR 2001k:20087 Zbl 0989.20030

[Cannon et al. 1996] J. W. Cannon, W. J. Floyd, and W. R. Parry, "Introductory notes on Richard Thompson's groups”, Enseign. Math. (2) 42:3-4 (1996), 215-256. MR 98g:20058 Zbl 0880.20027

[Cleary and Taback 2004] S. Cleary and J. Taback, "Combinatorial properties of Thompson's group F”, Trans. Amer. Math. Soc. 356:7 (2004), 2825-2849. MR 2005b:20074 Zbl 1065.20052

[Cleary and Taback 2005] S. Cleary and J. Taback, "Dead end words in lamplighter groups and other wreath products”, Q. J. Math. 56:2 (2005), 165-178. MR 2006h:20055 Zbl 02230593

[Fordham 2003] S. B. Fordham, "Minimal length elements of Thompson's group F", Geom. Dedicata 99 (2003), 179-220. MR 2004g:20045 Zbl 1039.20014

[Higman 1961] G. Higman, "Subgroups of finitely presented groups", Proc. Roy. Soc. Ser. A 262 (1961), 455-475. MR 24 \#A152 Zbl 0104.02101

[Ol'shanskiŭ 1997] A. Y. Ol'shanskiı̌, "On subgroup distortion in finitely presented groups", Mat. Sb. 188:11 (1997), 51-98. In Russian; translated in Sb. Math. 188 (1997), 1617-1664. MR 99a:20038 Zbl 0905.20020

Received January 28, 2005.

\author{
SEAN CLEARY \\ DEPARTMENT OF MATHEMATICS R8133 \\ The City College of NeW York \\ NEW York, NY 10031 \\ cleary@sci.ccny.cuny.edu \\ http://www.sci.ccny.cuny.edu/ cleary
}

